\title{
ON THE CONVERSE OF THE NONEXPANSIVE MAP FIXED POINT THEOREM FOR HILBERT SPACE
}

\author{
ROBERT SINE
}

\begin{abstract}
A simple proof is given of Ray's theorem that a nonempty, closed, convex, and unbounded set in Hilbert space admits a fixed point free nonexpansive map.
\end{abstract}

A map $T$ is nonexpansive on its domain $K$ if $\|T x-T y\| \leq\|x-y\|$ for all $x$ and $y$ in $K$. If $K$ is nonempty, closed, convex, and bounded in Hilbert space then each nonexpansive selfmap of $K$ has a fixed point. This surprising result was obtained in 1965 independently by Browder [1], Göhde [2], and Kirk [3]. It should be pointed out that while $T$ is continuous in the norm topology because of the assumed Lipschitz condition, it need not be continuous in the weak topology so it is not clear how the weak compactness of such a set $K$ can be exploited. If $L$ is the half-line $\{x: x \geq 0\}$ of the one-dimensional Hilbert space, then the map $T x=x+1$ is nonexpansive and is obviously fixed point free.

If $K$ is a nonempty, closed, convex, unbounded set in $H$ which contains a half-line $L$, then $K$ admits a fixed point free nonexpansive map. One simply uses the fact that there is a nearest point Chebyshev map of $K$ onto $L$ which is nonexpansive. Then one composes $T$ by following the Chebyshev map onto $L$ by the shift that was used above on that half-line.

That would be all there is to it if a nonempty, closed, convex, unbounded set always contained a half-line. But this is not the case as elementary examples will show. W. O. Ray [4] gave a proof of the theorem below using the fact (which he first established): If $K$ is nonempty, closed, convex, and unbounded and, in addition, contains the origin (a harmless normalization for this affine problem), then $H$ contains an orthonormal set $\left\{e_{n}: n \geq 1\right\}$ so that $e_{1}+\cdots+e_{n}$ is in $K$ for all $n$. A rather noncomputational proof will be given below based on well-known properties of Hilbert spaces.

THEOREM ( RAY). If $K$ is a nonempty, convex, closed, unbounded set in Hilbert space, then $K$ admits a fixed point free nonexpansive map.

PROOF. If a set in $H$ looks bounded, it is bounded. This means that if each continuous linear functional on $K$ is bounded, then $K$ is a bounded set. This fact is a well-known version of the Banach Steinhaus Theorem. Thus if $K$ is unbounded there is a continuous functional $f$ so that

$$
K_{n}=\{x \text { in } K: f(x) \geq n\}
$$

is a nonempty subset of $K$ for every $n \geq 1$. The sets $\left\{K_{n}\right\}$ are also closed and convex so there is a Chebyshev nearest point map $P_{n}$ from $H$ onto $K_{n}$. Here is the

Received by the editors March 25, 1986.

1980 Mathematics Subject Classification (1985 Revision). Primary 47H09, 47H10; Secondary $54 \mathrm{H} 25$. 
only point where we use the fact that the space is Hilbert space-Chebyshev maps in Hilbert space are always nonexpansive.

We now want to define $T$ by $T=\sum c_{n} P_{n}$, where we take $c_{n}>0$ and $\sum c_{n}=1$ to insure that $T$ is nonexpansive. A little more care is necessary, however, to insure that $T$ is defined! A poorman's Montel principle assures us that $T$ is defined (=convergent) if the sum converges at one single point. Let $x$ in $K$ be the single point. It is not difficult to see that $\left\{c_{n}\right\}$ can be chosen to satisfy, in addition to the requirements above,

$$
\sum c_{n}\left\|P_{n}(x)\right\|<\infty
$$

Then $T$ is well defined.

If $w$ were a fixed point of $T$, then

$$
w=\sum c_{n} P_{n}(w)
$$

Now if $w$ is in $K_{n}$, then $P_{k}(w)=w$ for $k \leq n$. And if $k>n$, then $f P_{k}(w)>f(w)$. Since $f$ is linear,

$$
f(w)=f\left(\sum c_{n} P_{n}(w)\right)=\sum c_{n} f P_{n}(w)
$$

From this it is clear no point of $K$ can be fixed under $T$.

\section{REFERENCES}

1. F. E. Browder, Nonexpansive nonlinear operators in a Banach space, Proc. Nat. Acad. Sci. U.S.A. 54 (1965), 1041-1044.

2. D. Göhde, Zum Prinzep der Kontractiven Abbildung, Math. Nachr. 30 (1965), 251-258.

3. W. A. Kirk, A fixed point theorem for mappings which do not increase distances, Amer. Math. Monthly 72 (1965), 1004-1006.

4. W. O. Ray, The fixed point property and unbounded sets in Hilbert space, Tran. Amer. Math. Soc. 258 (1980), 531-537.

DEPARTMENT OF MATHEMATICS, UNIVERSITY OF RHODE ISLAND, KINGSTON, RHODE ISLAND 02881 (Current address)

Department of Mathematics, Iowa State University, Ames, Iowa 50011 